\title{
Instantaneous Information Propagation in a Traffic Stream through Inter-Vehicle Communication
}

\author{
Wen-Long Jin *and Wilfred W. Recker ${ }^{\dagger}$
}

May 20, 2005

\begin{abstract}
The advancement of wireless communication technology has made possible the consideration of inter-vehicle communication as a foundation for developing decentralized advanced transportation information systems that would function as a sort of "internet on the road". In this paper, we discuss the reliability of inter-vehicle communication in a traffic stream, dependent on the distribution of equipped vehicles. With the assumption that information propagation is instantaneous compared to vehicle movements, the reliability is measured by the probability of success for information to travel beyond a location; stochastic models are presented for both uniform and general traffic streams. In the models, the traffic stream is divided into a series of cells based on the transmission range, the structure of possible most-forward-within-range communication chains is clarified, the probabilities for information to travel to and beyond a vehicle at a certain hop are computed regressively, and the lower bound of the absolute success rate for information to travel beyond a point is determined. Based on the models, we examine the performance of information propagation for different penetration rates, transmission ranges, and traffic scenarios that include gaps and shock waves. Finally, some implications and future extensions of this effort are discussed.
\end{abstract}

\section{Introduction}

Intelligent Transportation Systems (ITS) that incorporate advanced technologies have been purported to offer efficiencies in tackling traffic congestion. Among such systems are Advanced Transportation Information Systems (ATIS) that broadcast such real-time traffic information as travel times to travelers. Some systems have been actually placed successfully into operation, e.g., ADVANCE (Boyce et al., 1994); all rely on a centralized distributor of the information, such as a local Transportation Management Center. The rapid advance in available information technology, especially, the development of wireless communication technologies, now makes feasible the exploration of traffic information systems that decentralize the tasks of collecting and disseminating traffic information. A number of efforts are currently underway to investigate inter-vehicle communications (IVC) based on mobile ad hoc networking technology as a means of developing "internet on the road" (e.g. CarTALK 2000; FleetNet). However, to date, these efforts have focused more on the protocols and routing algorithms for information transmissions than on the application of the technology as the foundation for a decentralized, real-time traffic information system.

*Institute of Transportation Studies, University of California, 522 Social Science Tower, Irvine, CA 92697, USA. Email: wjin@uci.edu

${ }^{\dagger}$ Department of Civil and Environmental Engineering, Institute of Transportation Studies, University of California, 522 Social Science Tower, Irvine, CA 92697, USA. Email: wwrecker@uci.edu. Corresponding author 
In an effort to explore the advantages of IVC in developing ITS, researchers at the California Institute of Telecommunications and Information Technology and the Institute of Transportation Studies of the University of California, Irvine, have been engaged in a comprehensive research effort aimed at the development of an autonomous, self-organizing, transportation management, information, and control system. Called Autonet, the long-term goal of the effort is deployment of an autonomous, self-organizing information network for effective management of interactions among intelligently informed vehicles, roadways, stations and consumers (drivers). The Autonet concept proposes to use vehicle-to-vehicle and vehicle-to-infrastructure communications to leverage cooperative, vehicle-centric pervasive computing as a platform for transportation management. At its core, Autonet can be visualized as a bundle of services supporting an arbitrary collection of transportation management applications. These services are provided by accessing a decentralized collection of computer systems using a variety of protocols, which in turn are implemented on any number of physical networking architectures. Compared to centralized ATIS systems, a decentralized system like Autonet offers some significant advantages: (1) the IVC components of the system require no capital investment on the part of the transportation agencies, and can evolve to a full system gradually, once a threshold market penetration is achieved; (2) both the monetary and labor costs to build and operate the system are directly distributed to the users of the system; (3) the system is much more resilient to disruption, particularly in the event of disasters, when communications, management, and control are most important; (4) the system can be anchored to the Internet as a platform for additional applications. It also poses significant challenge compared to infrastructure-based networks: (1) the routing problem, which is already hard to solve in fixed networks, is substantially more complicated in a mobile ad-hoc network; (2) although rapid progress made toward higher data transmission rates based on the IEEE 802.11 standard for wireless local area networking (WLAN) has put the promise of truly mobile computing within reach, communications bandwidth remains a scarce resource in a mobile ad-hoc network;(3) the possibility of propagation of malicious misinformation and access to any particular traveler's origin-destination information can pose a serious security problem.

Technology issues undoubtedly will play an important role in an assessment of candidate protocols for a distributed traffic information system based on IVC; however, in this paper we focus on the feasibility analysis for an initial prototype deployment of the distributed, ad-hoc vehicular information system, instead of ad-hoc network technology itself. Specifically, we focus on a fundamental issue regarding to performance of such a system; i.e., how far can information be expected to propagate in a traffic system under certain traffic conditions, penetration rate of equipped cars, and transmission range of wireless units? By providing some general answers to this question, we hope to help guide specification of appropriate communication devices, routing protocols, and database management schemes that would be required in order to have the most important information collected and distributed, and to determine the range of applications for which a mobile, ad hoc traffic information system might be effective.

In previous work, (Hartenstein et al., 2001) simulated the process of information propagation using the cellular automaton model, presenting results on the probability that two equipped vehicles could establish connection through IVC under different traffic conditions and transmission ranges. In (Yang, 2003), IVC was simulated with Paramics, a microscopic traffic simulator, and the maximum information propagation distance was evaluated against transmission range and penetration rate for unidirectional roads, bidirectional roads, and road networks. In (Ziliaskopoulos and Zhang, 2003), propagation of travel time information was studied in a grid road network through information exchange between vehicles traveling in opposite directions. In this case, the primary mechanism for information distribution is based on the flow of traffic, rather than relying on information hops among neighboring vehicles. This type of information propagation may be particularly important in the early stage of deployment when the penetration rate may be too low to support the "hopping" of information along a platoon of vehicles traveling in the same direction. However, under such 
conditions, an IVC-equipped vehicle would likely have to wait for another such equipped vehicle traveling in the opposite direction to pass within its communication range. Thus, such information propagation may suffer from high delay and may fail if vehicles in the other direction run into a queue.

In this paper, we are interested in information propagation through "multi-hopping" along a platoon of vehicles. However, unlike the simulation studies of (Hartenstein et al., 2001; Yang, 2003), we develop an analytical model of the probability that a message can travel beyond a point in a traffic stream on a link, either uni- or bi-directional. As shown in (Briesemeister et al., 2000), it only takes $110 \mathrm{~ms}$ to transmit 73 bytes with a rate at $3.6 \mathrm{~kb} / \mathrm{s}$. This means that, with an achievable transmission rate of $1 \mathrm{Mb} / \mathrm{s}$ or with a short message about traffic conditions, information exchange can be expected to be completed in a very short time, during which the movement of vehicles is inconsequential. In this sense, we consider the traffic stream as static, as compared to the information propagation, and we do not consider a dynamic communication topology (Rudack et al., 2002). That is, at any moment, we take a "snapshot" of the traffic stream and analyze information propagation in the vehicle network; we refer to this scenario as instantaneous information propagation. Note that, with the instantaneity assumption, there is no distinction between information exchange between vehicles traveling in opposite directions and that between vehicles traveling in the same direction; i.e., either have equivalent model representations relative to information propagation. Further, since we are interested in establishing the maximum expected performance of information propagation, we assume that information propagates in a manner of "most forwarded within range" (MFR) (Takagi and Kleinrock, 1984); i.e., a message is transmitted to the farthest equipped vehicle within the transmission range. With this assumption, a vehicle at a hop can establish communication with at most one at the next hop and at most one at the preceding hop. In this paper, notations in Table 1 are used.

The remainder of the paper is organized as follows. First, we model information propagation for uniform traffic and analyze possible communication chains by the success rate for information to travel beyond a point (Section 2). Then, we evaluate the performance of IVC in uniform traffic in terms of transmission range, traffic density, and penetration rate (Section 3). In Section 4, we present a stochastic model of information propagation in general traffic and study properties of the model for a randomly distributed traffic stream. With the model, we study information propagation in a traffic stream with a gap or a shock wave in Section 5. Finally, we remark on the results in the conclusion section.

\section{A stochastic model of information propagation in uniform traffic}

In this section, we consider on an infinitely long road a uniform stream of traffic, whose density is $\rho$ and speed $v$. We assume that the communication range, $R$, is constant for each hop. We assume that there are $n$ vehicles inside a transmission range $R$; i.e., traffic density for all lanes is $\rho=n / R$. Further, we assume the penetration rate of equipped vehicles as $\mu(0 \leq \mu \leq 1)$. Thus the probability of a vehicle to be equipped is $\mu$, that of a vehicle not to be equipped as $\nu \equiv 1-\mu$, and the distribution of independent equipped cars forms Bernoulli trials (Feller, 1950, Chapter VI).

\subsection{Communication chains}

The performance of information propagation in a uniform traffic stream can be measured by the success rate for information to travel beyond a point in the traffic stream. This point is determined by the unique sequence of informed vehicles, starting from the information source, that form the set of MFR vehicles. Such a sequence is called an MFR communication chain, or simply a communication 
chain. In a communication chain, an informed vehicle is called a node, and the connection between two consecutive nodes a hop. The number of hops in a chain is stochastic, dependent on the distribution of equipped vehicles in the traffic stream. In this communication chain, we denote the information source by node 0 , and any other node by the number of hops $h$ for information to reach it; the location of node $h$ in the traffic stream is designated by $x(h)$.

In uniform traffic, information is limited to travel only as far as $h R$ within $h$ hops. Starting from the information source, we divide the traffic stream into a number of cells in the direction of information propagation, with the length of each cell equal to the communication range $R$. Within cell $c(c=1, \cdots)$, vehicle $k(k=1, \cdots, n)$ is denoted by $(c, k)$. So, $x(d, j)>x(c, k)$ either when $d>c$ or when $d=c$ and $j>k$, where $x(c, k)$ is the position of vehicle $(c, k)$. Under the assumption of uniform traffic, we have $x(c, n)=c R$ and $x(c+1, k)-x(c, k)=R$. Note that the information source is not inside cell 1 .

The event that vehicle $(c, k)$ is node $h$ on a chain is denoted by $(c, k ; h)$, and the probability of the event by $P(c, k ; h)$. Therefore, $P(c, k ; h)$ is equal to the probability for information to travel to $(c, k)$ in $h$ hops. In order to compute $P(c, k ; h)$, we first study the properties of communication chains within the uniform traffic stream.

Theorem 2.1 (Regulation of nodes) From the assumption of MFR and the limit of communication range, we can derive the following properties of nodes for a communication chain.

1. If vehicle $(c, k)$ is node $h(h \geq 1)$, then vehicle $(c, k)$ is equipped and no vehicles between $x(h)$ and $x(h-1)+R$ are equipped. That is, node $h$ is the farthest equipped vehicle in the transmission range of node $h-1$.

2. There are at most two nodes in a cell.

3. If there is a node in cell $c$, there is at least one node in cell $c-1$.

4. In cell 1 , if vehicle $(1, k)$ is the farthest equipped within the transmission range of the information source, it gets information in one hop. That is, if vehicle $(1, k)$ is on a communication chain, it is node 1.

5. At hop $h$, a message travels at most to cell $h$. That is, node $h$ of a chain is at most in cell $h$. In this case, node $i$ is in cell $i$ for $i=1, \cdots, h-1$.

6. At hop $h$, a message travels at least to cell $\left[\frac{(h+1)}{2}\right]$, where function $[x]$ returns the smallest integer that is bigger than or equal to $x$. That is, node $h$ of a chain is at least in cell $\left[\frac{(h+1)}{2}\right]$. In this case, node $i$ is in cell $\left[\frac{(i+1)}{2}\right]$ for $i=1, \cdots, h-1$.

In the above, if nodes $h-1$ and $h$ belong to the same cell, we then refer to hop $h$, from node $h-1$ to $h$, as an intra-cell hop, and as an inter-cell hop otherwise. If for example, vehicle $(2,2)$ is the MFR in cell 2, it gets informed at hop 2 through an inter-cell hop when, e.g., $(1,2)$ is informed. If, however, the MFR vehicle in cell 1 is $(1,1)$, vehicle $(2,2)$ can get informed only when (i) vehicle $(2,1)$ is equipped and (ii) vehicle $(3,1)$ is not, taking three hops for information to reach $(2,2)$, with the last hop being an intra-cell hop.

Theorem 2.2 (Regulation of hops) From MFR or Theorem 2.1, we have the following properties for hops in a communication chain.

1. The first vehicle in a cell can not be the end of an intra-cell hop.

2. There exist no consecutive intra-cell hops. 
3. The initial hop, i.e., hop 1, is an inter-cell hop.

4. The minimum number of hops from a node in cell $c(c \geq 2)$ to a node in cell $c+1$ is one (with an inter-cell hop), and the maximum number is three (an intra-cell hop, followed consecutively by an inter-cell hop and an intra-cell hop). Information propagation from a node in cell $c$ (c $\geq 2)$ to a node in cell $c+1$ by two hops can occur as an intra-cell hop followed by a inter-cell hop or vice versa.

5. The minimum number of hops from node 0 to a node in cell $c(c \geq 1)$ is $c$ (with all inter-cell hops), and the maximum number is $2 c-1$ via a pattern of an initial inter-cell hop followed by $c-1$ consecutive combinations of inter-cell hop plus intra-cell hop.

From property 4 of Theorem 2.2, if we select any particular node in each cell from a communication chain (the information source and the last node have to be selected), the communication chain can be broken into sub-chains at these nodes that comprise one of four types of sub-chains having one or more hops: (i) an inter-cell hop, (ii) an inter-cell hop followed by an intra-cell hop, (iii) an intra-cell hop followed by an inter-cell hop, (iv) an intra-cell hop followed by an inter-cell hop and an intra-cell hop.

Theorem 2.3 (Properties of sub-chains) We have the following properties of sub-chains.

1. An arbitrary communication chain cannot be constructed from only one type of sub-chain.

2. Any communication chain can be broken into a unique sequence of types $i$ and ii sub-chains.

3. An arbitrary communication chain cannot be constructed from combinations of any other two sub-chains.

4. Types $i$ and ii sub-chains are independent for the structure of a communication chain since any two communication chains spanning in c cells are different in structure from each other if their last sub-chains are different. Therefore, there are $2^{c-1}$ different structures for a communication chain spanning in cells.

Therefore, type i sub-chain, called a one-hop jump, and type ii sub-chain, called a two-hop jump, form the minimum basis of all possible communication chains. That is, an arbitrary communication chain can be constructed from them, and different sequences of them form different communication chains.

\subsection{Information propagation in uniform traffic}

To compute the probability for vehicle $(c, k)$ to be node $h$ of all possible communication chains, $P(c, k ; h)$, we first consider the different structures a communication chain can take, determined by the sequence of one-hop and two-hop jumps, and then consider the probability for each structure. In addition, we define the joint probability of $(c, i ; h)$ and $(c, l ; h-1)(1 \leq l<i)$ by $\mathcal{P}(c, l, i ; h)$. We compute $P(c, k ; h)$ regressively as follows.

In cell $1, h=1$, and $P(1, k ; 1)$ equals to the probability that vehicle $(1, k)$ is the farthest equipped vehicle. Thus we have

$$
P(1, k ; 1)=\mu \nu^{n-k} .
$$

Note that the probability that there is no equipped car in cell 1 is $\nu^{n}$. In addition,

$$
\mathcal{P}(1, l, i ; 1)=0
$$


for any $1 \leq l<i \leq n$.

For cell $c$, we assume that $P(c, k ; h)$ is known for $k=1, \cdots, n$ and $h=c, \cdots, 2 c-1$. Then, we consider the probability for vehicle $(c+1, k)(k=1, \cdots, n)$ to be node $h+1$ on a communication chain. From Theorem 2.3, $(c+1, k)$ can belong to one of two mutually exclusive, exhaustive types of chains; i.e., it can be the end of a one-hop jump from node $h$ in cell $c$ or the end of a two-hop jump from node $h-1$ in cell $c$.

If node $(c+1, k ; h+1)$ is connected to node $(c, i ; h)$ through a one-hop jump, the following conditions have to be satisfied: (i) vehicle $(c, i)$ is node $h$ on the communication chain, where $h$ can be between $c$ and $2 c-1$; (ii) $x(c+1, k)-x(c, i) \leq R$; i.e., $i$ can vary from $k$ to $n$; (iii) vehicle $(c+1, k)$ is equipped; (iv) vehicles $(c+1, k+1)$ to $(c+1, i)$ are not equipped according to MFR; and (v) none of vehicles $(c, k)$ to $(c, i-1)$ can be node $h-1$. Therefore, the probability for node $(c+1, k ; h+1)$ to be connected from node $h$ in cell $c$ through a one-hop jump is given by

$$
P_{1}(c+1, k ; h+1)=\sum_{i=k}^{n}\left(P(c, i ; h)-\sum_{l=k}^{i-1} \mathcal{P}(c, l, i ; h)\right) \mu \nu^{i-k},
$$

where $k=1, \cdots, n ; h=c, \cdots, 2 c-1$.

If node $(c+1, k ; h+1)(k=2, \cdots, n)$ is connected to node $(c, i ; h-1)$ through a two-hop jump, with an intermediate node $(c+1, j ; h)$, the following conditions have to be satisfied: (i) vehicle $(c, i)$ is node $h-1$ on the communication chain, where $h-1$ can be between $c$ and $2 c-1$; (ii) $x(c+1, k)-x(c, i)>R$; i.e., $i$ can vary from 1 to $k-1$; (iii) vehicle $(c+1, j)$ is equipped, where $1 \leq j \leq i$; (iv) vehicles $(c+1, j+1)$ to $(c+1, i)$ are not equipped according to MFR; (v) vehicle $(c+1, k)$ is equipped; (vi) vehicles $(c+1, k+1)$ to $(c+2, j)$ are not equipped according to MFR; and (vii) none of vehicles $(c, j)$ to $(c, i-1)$ can be node $h-2$. Therefore, the probability for node $(c+1, k ; h+1)$ to be connected from node $h-1$ in cell $c$ through a two-hop jump is given by

$$
\begin{aligned}
P_{2}(c+1, k ; h+1) & =\sum_{i=1}^{k-1} \sum_{j=1}^{i}\left(P(c, i ; h-1)-\sum_{l=j}^{i-1} \mathcal{P}(c, l, i ; h-1)\right) \mu \nu^{i-j} \mu \nu^{n-k+j} \\
& =\sum_{i=1}^{k-1} \sum_{j=1}^{i}\left(P(c, i ; h-1)-\sum_{l=j}^{i-1} \mathcal{P}(c, l, i ; h-1)\right) \mu^{2} \nu^{n+i-k},
\end{aligned}
$$

where $k=2, \cdots, n ; h-1=c, \cdots, 2 c-1$. Similar to the analysis above, we can find the joint probability for $(c+1, k ; h+1)$ and $(c+1, j ; h)(k=2, \cdots, n, j=1, \cdots, k-1)$

$$
\mathcal{P}(c+1, j, k ; h+1)=\sum_{i=j}^{k-1}\left(P(c, i ; h-1)-\sum_{l=j}^{i-1} \mathcal{P}(c, l, i ; h-1)\right) \mu^{2} \nu^{n+i-k} .
$$

Note that, when $k=1$, we only have $(3)$ or $P_{2}(c+1,1 ; h+1)=0$, and, when $h=2 c$, we only have (4) or $P_{1}(c+1, k ; 2 c+1)=0$ since $P(c, i ; 2 c)$ does not exist. Since a one-hop jump and two-hop jump are mutually exclusive and exhaustive in forming communication chains, we obtain $P(c+1, k ; h)$ for $k=1, \cdots, n$ and $h=c+1, \cdots, 2 c+1$ as

$$
P(c+1, k ; h+1)=P_{1}(c+1, k ; h+1)+P_{2}(c+1, k ; h+1),
$$

where $P_{1}$ and $P_{2}$ are computed respectively from (3) and (4). For the purpose of generalization, we define $P(c, k ; h)=0$ when $h<c$ or $h>2 c-1$.

In the case when a communication chain consists only of one-hop jumps; i.e., when we omit cases with intra-cell communications, we have a model for restricted information propagation

$$
P(h+1, k ; h+1)=\sum_{i=k}^{n} P(h, i ; h) \mu \nu^{i-k},
$$


from which we can see that, for this restricted case, $P(h+1, k ; h+1)$ actually is a convolution of $P(h, k ; h)$ with a geometric binomial distribution. This results in a negative binomial distribution or Pascal distribution (Feller, 1950, Section VI.8), which can be written as

$$
P(h, k ; h)=\left(\begin{array}{c}
h+n-k-1 \\
n-k
\end{array}\right) \mu^{h} \nu^{n-k} .
$$

Note that, since this model does not consider communication chains with two-hop jumps, it is not complete and cannot be used in applications.

In general, when considering both one-hop and two-hop jumps, we cannot obtain $P(c, k ; h)$ in closed form. To obtain the aggregate performance measurement of an IVC system, we first define the cumulative probability, or the success rate for information to travel beyond vehicle $(c, k)$ at $h$ hops, by

$$
S(c, k ; h)=\sum_{(d, i)=(c, k)}^{(h, n)} P(d, i ; h),
$$

where $\frac{(h+1)}{2} \leq c \leq h$.

The absolute success rate for information to travel beyond $(c, k)$, regardless of the number of hops, can not be smaller than $S(c, k ; h)$ for any $h$. Therefore, we have a lower bound of the absolute success rate as

$$
s(c, k)=\max _{h} S(c, k ; h) .
$$

Note that, theoretically, the domain of $h$ is $[1, \infty)$. However, in computation, we can obtain the maximum for sufficiently large $h$.

The algorithm for computing all performance measurements in IVC is given in Table 2. Although there is a subtle difference between the actual success rate and its lower bound $(s(1,1)$ is accurate, and the difference for other locations is expected not to be significant), knowing the lower bound is useful in practical applications. In the results that follow, we use $s(c, k)$ as a measurement of the performance of information propagation. Further, we denote the maximum $x(c, k)$ for which $s(c, k)=s$ as the $s$ information front, and the distance between the $s$ information front and information source as the $s$ information progress.

\section{Analysis of information propagation in uniform traffic}

In this section, we employ the relationships developed in the previous section to evaluate the performance of IVC in uniform traffic in terms of transmission range, traffic density, and penetration rate.

\subsection{Information propagation success rate for different hops}

In Figure $1, S(c, k ; h)$ and $s(c, k)$ is plotted for first ten hops for $\mu=0.05$ and $n=100$; here the $x$-axis is defined in terms of $(c-1) n+k$ segments, where $(c-1) n+n=c n$ represents the limiting value for the farthest vehicle (counted from the information source) that could possibly receive the information in $h$ hops. The results demonstrate that, as expected, the success rate for information to travel a distance equal to a particular number of cells generally decreases with the number of hops involved; an exception to this is noted for distances within the vicinity of the maximum distance

for a given number of hops. For example, the success rate for information reach beyond vehicle 
$(c, k)=200$ at four hops is above $90 \%$ while with five hops it is below $90 \%$; however, the success rate for information reach beyond vehicle $(c, k)=300$ at four hops is about $77 \%$ while with five hops it remains only slightly below $90 \%$. From the figure, we can also see that $s(c, k)$ is non-increasing and piece-wise.

\subsection{Minimum number of vehicles for information propagation}

In this subsection, we compute the minimum number of vehicles in a cell that is required, for a given penetration rate, for information to travel a number of hops with a desired success rate. For hop 1, for example, we can compute the required minimum number of vehicles, $n$, exactly by $n \geq \frac{\ln (1-s)}{\ln \nu}$, from $S(1,1 ; 1)=1-\nu^{n} \geq s$.

The minimum numbers of vehicles required to achieve $90 \%$ success rate for first ten hops under different penetration rates are listed in Table 3. From the table, we can draw the following conclusions. First, for the same penetration rate, the minimum number of required vehicles increases with hops. This is simply because a communication chain with more nodes is harder to form than with fewer. Second, for the same number of hops, the minimum number of required vehicles decreases with the penetration rate, as expected.

For illustration, the minimum numbers of vehicles required to achieve success rates from $98 \%$ to $80 \%$ for a penetration rate $\mu=10 \%$ are listed in Table 4 for the first 10 hops. From this table, we can see that the minimum number of required vehicles decreases with the desired success rates, as expected.

In addition to the cases described in the two tables above, we can compute the required minimum number of vehicles in a cell for any given success rate and penetration rate from (6). Since the number of vehicles in a cell is equal to the traffic density times the transmission range, the model (6) can be used to determine the requirement for different traffic conditions and transmission ranges, given a penetration rate and desired success rate.

\subsection{Information propagation for different traffic densities and transmis- sion ranges}

In this subsection, the performance of information propagation, measured by the lower bound of absolute success rate $s(c, k)$, is analyzed as a function of traffic density and transmission range.

As an example, we consider free flow on a four-lane road with traffic density at $\rho=14$ veh $/ \mathrm{km} /$ lane. We assume uniform spacing of vehicles in any lane, and that vehicles in adjacent lanes are staggered such that the distance between two nearest vehicles is about $18 \mathrm{~m}^{1}$, or that the distance between two nearest vehicles in the same lane is about $71 \mathrm{~m}$. Therefore, the number of vehicles within a transmission range $R$ (unit: $\mathrm{km}$ ) is given by $n=56 R$. Under these conditions, the success rate for distances up to $10 \mathrm{~km}$ is shown in Figure 2, for $R=1.0,0.5,0.2,0.1 \mathrm{~km}$. This figure tells us that, as expected, the larger transmission range, the farther information propagates. For $R=1.0 \mathrm{~km}$, information can travel beyond $5.7 \mathrm{~km}$ with $90 \%$ success rate.

For the same traffic system as above but with lower density at $\rho=5 \mathrm{veh} / \mathrm{km} / \mathrm{lane}$, the number of vehicles inside a transmission range is $n=20 R$. In this traffic stream, the distance between two nearest vehicle on the same lane is $200 \mathrm{~m}$. Success rates for the same set of transmission ranges as in the preceding example are shown in Figure 3. For transmission range of $1 \mathrm{~km}$, information can only travel for about $0.3 \mathrm{~km}$ with $80 \%$ success rate. In these two examples, traffic is relatively sparse. In cases where traffic density per lane is close to the jam density, say about $150 \mathrm{veh} / \mathrm{km} / \mathrm{lane}$, as one can estimate from Tables 3 and 4 that information can travel significantly farther.

\footnotetext{
${ }^{1}$ This amounts to the most ideal spacing for transmission success and, for purposes of information exchange, is equivalent to assuming a traffic density at $\rho=56 \mathrm{veh} / \mathrm{km} /$ lane for a single-lane highway
} 


\section{A stochastic model of instantaneous information propaga- tion in general traffic}

In this section, we extend the model to the case of instantaneous information propagation in general traffic.

\subsection{A stochastic model for information propagation in general traffic}

Under conditions of uniform traffic in the previous examples, the length of a cell was identically equal to the communication range $R$, and the number of vehicles within each cell was constant $R \rho$. The situation for the case of general (non-uniform) traffic can be represented as shown in Figure 4, in which the position of each vehicle in the traffic stream is identified by a (non-unique) number that represents its cell membership. In this traffic stream, the information source is at car 0 , cars labeled with a 1 are in cell 1, and so on. For this traffic stream, cells can be determined as follows. First, within $R$ from the information source, we find the farthest car, whose position is the end of cell 1. Then, within $R$ from the farthest car in cell 1, we find the farthest car in cell 2 . We continue this process for the whole traffic stream. Due to the non-uniform distribution of vehicles, as shown in the figure, the length of a cell is less than or equal to the transmission range $R$. When determining cells, we can also obtain the number of vehicles in cell $c$ and denote it by $n_{c}$. Assuming that no two vehicles have the same location (even on a multi-lane road), we then can order vehicles in cell $c$ from 1 (the closest) to $n_{c}$ (the farthest) and refer to the $k$ th vehicle in cell $c$ by $(c, k)$. If the location of vehicle $(c, k)$ is $x(c, k), x\left(c_{1}, k_{1}\right) \neq x\left(c_{2}, k_{2}\right)$ if $\left(c_{1}, k_{1}\right) \neq\left(c_{2}, k_{2}\right)$.

Further, for a vehicle $(c, k)(c>1)$, we can find vehicle $(c-1, \underline{k})$ such that $\underline{k}$ is the minimum satisfying

$$
|x(c, k)-x(c-1, \underline{k})| \leq R .
$$

That is, $(c-1, \underline{k})$ is the farthest, upstream (in the direction of information propagation) car inside the transmission range of $(c, k)$. Similarly, we can find the farthest, downstream car $(c+1, \bar{k})$ such that $\bar{k}$ is the maximum satisfying

$$
|x(c+1, \bar{k})-x(c, k)| \leq R,
$$

where $c \geq 1$. Note that, in uniform traffic, $\underline{k}=k$, and $\bar{k}=k$.

We continue to define $P(c, k ; h)$ as the probability for information to reach car $(c, k)$ at hop $h$, under MFR. Since the properties of communication chains are the same as those in uniform traffic, we can derive the stochastic model using the same approach as in Subsection 2.2. For the first hop, when $h=c=1$, we still have a geometric distribution, $P(1, k ; 1)=\mu \nu^{n_{1}-k}$, for $k=1, \cdots, n_{1}$, and all joint probabilities are zero.

In a regressive manner, we assume that $P(c, k ; h)$ is known for $k=1, \cdots, n_{c}$ and $h=c, \cdots, 2 c-1$ and then derive a recursive formula for $P(c+1, k ; h)$ for $k=1, \cdots, n_{c+1}$ and $h=c+1, \cdots, 2 c+1$ as follows.

If node $(c+1, k ; h+1)$ is connected to node $(c, i ; h)$ through a one-hop jump, the following conditions have to be satisfied: (i) vehicle $(c, i)$ is node $h$ on the communication chain, where $h$ can be between $c$ and $2 c-1$; (ii) $x(c+1, k)-x(c, i) \leq R$; i.e., $i$ can vary from $\underline{k}$ to $n_{c}$; (iii) vehicle $(c+1, k)$ is equipped; (iv) vehicles $(c+1, k+1)$ to $(c+1, \bar{i})$ are not equipped according to MFR; and $(\mathrm{v})$ none of vehicles $(c, \underline{k})$ to $(c, i-1)$ can be node $h-1$. Therefore, the probability for node $(c+1, k ; h+1)$ to be connected from node $h$ in cell $c$ through a one-hop jump is given by

$$
P_{1}(c+1, k ; h+1)=\sum_{i=\underline{k}}^{n_{c}}\left(P(c, i ; h)-\sum_{l=\underline{k}}^{i-1} \mathcal{P}(c, l, i ; h)\right) \mu \nu^{\bar{i}-k},
$$


where $k=1, \cdots, n_{c+1} ; h=c, \cdots, 2 c-1$.

If node $(c+1, k ; h+1)\left(k=2, \cdots, n_{c+1}\right)$ is connected to node $(c, i ; h-1)$ through a twohop jump, with an intermediate node $(c+1, j ; h)$, the following conditions have to be satisfied: (i) vehicle $(c, i)$ is node $h-1$ on the communication chain, where $h-1$ can be between $c$ and $2 c-1$; (ii) $x(c+1, k)-x(c, i)>R$; i.e., $i$ can vary from 1 to $\underline{k}-1$; (iii) vehicle $(c+1, j)$ is equipped, where $1 \leq j \leq \bar{i}$; (iv) vehicles $(c+1, j+1)$ to $(c+1, \bar{i})$ are not equipped according to MFR; (v) vehicle $(c+1, k)$ is equipped; (vi) vehicles $(c+1, k+1)$ to $(c+2, \bar{j})$ are not equipped according to MFR; and (vii) none of vehicles $(c, \underline{j})$ to $(c, i-1)$ can be node $h-2$. Therefore, the probability for node $(c+1, k ; h+1)$ to be connected from node $h-1$ in cell $c$ through a two-hop jump is given by

$$
\begin{aligned}
P_{2}(c+1, k ; h+1) & =\sum_{i=1}^{k-1} \sum_{j=1}^{\bar{i}}\left(P(c, i ; h-1)-\sum_{l=\underline{j}}^{i-1} \mathcal{P}(c, l, i ; h-1)\right) \mu \nu^{\bar{i}-j} \mu \nu^{n_{c+1}-k+\bar{j}} \\
& =\sum_{i=1}^{k-1} \sum_{j=1}^{\bar{i}}\left(P(c, i ; h-1)-\sum_{l=\underline{j}}^{i-1} \mathcal{P}(c, l, i ; h-1)\right) \mu^{2} \nu^{\bar{i}-j+n_{c+1}-k+\bar{j}}
\end{aligned}
$$

where $k=2, \cdots, n_{c+1} ; h-1=c, \cdots, 2 c-1$. Similarly, we can update the joint probability for $(c+1, k ; h+1)$ and $(c+1, j ; h)\left(k=2, \cdots, n_{c+1}, j=1, \cdots, k-1\right)$

$$
\mathcal{P}(c+1, j, k ; h+1)=\sum_{i=\underline{j}}^{\underline{k}-1}\left(P(c, i ; h-1)-\sum_{l=\underline{j}}^{i-1} \mathcal{P}(c, l, i ; h-1)\right) \mu^{2} \nu^{\bar{i}-j+n_{c+1}-k+\bar{j}} .
$$

We then obtain $P(c+1, k ; h)$ for $k=1, \cdots, n_{c+1}$ and $h=c+1, \cdots, 2 c+1$,

$$
P(c+1, k ; h+1)=P_{1}(c+1, k ; h+1)+P_{2}(c+1, k ; h+1),
$$

where $P_{1}$ and $P_{2}$ are computed respectively from (12) and (13).

We define $P(c, k ; h)$ as 0 for $h<c$ or $h>2 c-1$. Then, $S(c, k ; h)=\sum_{(d, i)=(c, k)}^{(h, n)} P(d, i ; h)$ and $S(c, k ; h)=0$ when $h<c$. That is, $S(c, k ; h)$ is the probability that information can travel beyond $(c, k)$ at $h$ hops. We then define (the lower bound of) the absolute success rate for information to travel to or beyond $(c, k)$, regardless of the number of hops, as $s(c, k)=\max _{h} S(c, k ; h)$.

\subsection{Properties of the model for random traffic streams}

For illustration, we consider a traffic stream with a length equal to fifteen times of transmission range $R$. Inside each range segment, the number of vehicles is assumed to be a uniform random variable between 75 and 125, and vehicle positions are also assumed to be uniformly distributed. Note that the actual length of a cell is usually smaller than the transmission range, and that the number of vehicles inside each cell is different from that randomly generated.

The success rates of first ten hops in the first ten cells with respect to vehicle number are shown in Figure 5 for the traffic stream above and for a penetration rate $\mu=5 \%$. In the figure, the curve for the first hop is the same as for uniform traffic, but those for later hops are not as smooth due to the non-uniform distribution of vehicles, as characterized by the discrepancies both between $k$ and $\underline{k}$, and between $k$ and $\bar{k}$. Figure 6 shows the success rates with respect to vehicle position; these curves are even less smooth. In both figures, the dotted envelope curves are the lower bounds of the absolute success rates. The non-smoothness is expected to increase for less regular (i.e., higher variance) distributions of traffic and/or sparser traffic. 


\section{Examples for instantaneous information propagation in gen- eral traffic}

\subsection{Information propagation through a gap}

In this subsection, we consider two uniform traffic streams at $\rho=56 \mathrm{veh} / \mathrm{km}$ with a gap of $g \mathrm{~km}$ in between. For the purpose of simplification, we assume that one stream ranges from $-\infty$ to 0 , and the other from $g$ to $\infty$ and set the penetration rate as $\mu=10 \%$ and transmission range as $R=1$ $\mathrm{km}$. Note that the conditions are similar to those in Figure 2.

The success rates of information propagation for $g=0.4 \mathrm{~km}$ for different locations of information source are shown in Figure 7. The figure shows that the success rates drop significantly upstream of the gap; the magnitude of the drops for different locations of the information source are comparable. When the information source is at $x=0$, we observe that the success rate at the first hop is much smaller than that of the other cases, since there are just three fifths vehicles in the first cell.

For different sizes of a gap ranging from 0 to $1 \mathrm{~km}$, Figure 8 describes the $90 \%$ information progress for different locations of information source, ranging from $-9 \mathrm{~km}$ to $1 \mathrm{~km}$ in increments of 0.5 $\mathrm{km}$. From the figure, the following can be observed. First, as expected, information can propagate to its full distance of about $5.71 \mathrm{~km}$ at a $90 \%$ confidence when the source is to the downstream of the gap, or when there is no gap. Second, this progress decreases to 5.66 when the information source is at $-7 \mathrm{~km}$ and the gap size is of $0.1 \mathrm{~km}$. This is due to the fact that the gap in cell 8 affects the success rate at hop 8 , which contributes to the absolute success rate in cell 6 , where success rate reaches $90 \%$ at $5.66 \mathrm{~km}$. We can see that, when the information source is further upstream from the gap, the $90 \%$ progress will not be affected. Third, information progress for different locations of information source is a non-increasing function of gap size. Fourth, the progress first decreases when the gap is closer to the information and then rebounds when it is even closer. In addition, we observe some local oscillations, e.g., for gap size of $0.1 \mathrm{~km}$ when information source is between $-6 \mathrm{~km}$ and $-4 \mathrm{~km}$. These phenomena suggest that the effect of a gap on information propagation is quite nonlinear and should be treated specifically.

\subsection{Information propagation through a shock wave}

In this subsection, we consider information propagation on an infinitely long road within a stream of traffic subjected to a shock wave. It is generally accepted that, for uninterrupted traffic flow, there is a density-speed relationship, $v=V(\rho)$ (Hall et al., 1986). In this example, we assume the so-called triangular fundamental diagram (Munjal et al., 1971; Newell, 1993),

$$
V(\rho)= \begin{cases}v_{f}, & 0 \leq \rho \leq \rho_{c} \\ \frac{\rho_{c}}{\rho_{j}-\rho_{c}} \frac{\rho_{j}-\rho}{\rho} v_{f}, & \rho_{c}<\rho \leq \rho_{j}\end{cases}
$$

for conditions in which $v_{f}=104 \mathrm{~km} / \mathrm{h}$ is the free flow speed, $\rho_{j}=150 \mathrm{veh} / \mathrm{km} /$ lane the jam density, and $\rho_{c}=0.2 \rho_{j}=30 \mathrm{veh} / \mathrm{km} /$ lane the critical density where flow-rate, $q=\rho v$, attains its maximum, i.e. the capacity.

Based on the triangular diagram, (16), we examine information propagation on a unidirectional road of two homogeneous lanes of traffic. We assume that initially we have capacity flow with $\rho_{-}=30$ veh $/ \mathrm{km} /$ lane for traffic upstream to $x=0$ and congested flow $\rho_{+}=40 \mathrm{veh} / \mathrm{km} /$ lane for downstream traffic. From the fundamental diagram, we then have the corresponding speeds at $v_{-}=104 \mathrm{~km} / \mathrm{h}$ and $v_{+}=97 \mathrm{~km} / \mathrm{h}$. Under these conditions, a shock wave forms and travels backward at speed $v_{s}=-26 \mathrm{~km} / \mathrm{h}$. Further, we assume that $\mu=10 \%$ and $R=1 \mathrm{~km}$. If initially information is carried by a vehicle at $x_{0}<0$, the vehicle will cross the shock wave at time $t_{c}=\left|x_{0}\right| /\left(v_{-}-v_{s}\right)$. 
In Figure 9, we show the success rates of information propagation in both forward and backward directions for four instants of time: $t_{0}=0, t_{1}=2.3 \mathrm{~min}, t_{2}=4.6 \mathrm{~min}$, and $t_{3}=9.9 \mathrm{~min}$. The corresponding locations of information source are $-10 \mathrm{~km},-6 \mathrm{~km},-2 \mathrm{~km}$, and $6.5 \mathrm{~km}$, and the locations of shock wave are $0,-1 \mathrm{~km},-2 \mathrm{~km}$, and $-4.3 \mathrm{~km}$. Note that the information source meets the shock wave at $t_{2}$. Then at these four time instants, we take a snapshot of the traffic stream and compute the lower bound of the absolute success rate $s(c, k)$ for both forward and backward information propagation. From the four curves, we can see that the slope of the forward branches at $t_{0}$ and $t_{1}$ becomes smaller after meeting shock wave. This is as expected since the downstream of the shock wave has higher density. In the backward branches at $t_{3}$, the slope becomes larger upstream of the shock.

In Figure 10, we show the $95 \%$ information front in both forward and backward directions. In this figure, the curve for information source is almost a straight line owing to the slight difference in traffic conditions on both sides of the shock wave. However, we can see the dramatic change in both forward and backward information front. In the forward information propagation, the curve of $95 \%$ information progress first keeps a constant distance with the information source, and the distance is about $4.0 \mathrm{~km}$, which is the information progress in the upstream (sparser) traffic. Then at about $2.0 \mathrm{~min}$, the profile meets the shock wave, and the downstream (denser) traffic starts to contribute to the forward information propagation. From then on, the curve moves forward much faster than the information source. Then, at $4.6 \mathrm{~min}$, the source crosses the shock wave, and the denser traffic is the sole contributor to forward information propagation, and, for the rest of the time, the curve maintains a constant distance, $27.1 \mathrm{~km}$, from the source. Thus we can see that the small difference in traffic density has a huge influence on information propagation.

The backward information progress curve follows a similar pattern in its distance relative to the source, but the change is more gradual. In addition, the backward curve crosses the shock wave at $13.0 \mathrm{~min}$, and the message is not able to pass the shock wave after $13.0 \mathrm{~min}$. Note that, at 13.0 min, the source is at $11.6 \mathrm{~km}$, the backward $95 \%$ information front is at $-5.6 \mathrm{~km}$, and their distance is smaller than the information progress in the denser traffic.

\section{Discussion}

In this paper, we analyzed information propagation in a traffic stream via inter-vehicle communication, which is assumed to be instantaneous compared to vehicle movements. We proposed a stochastic model for computing the success rate for information to travel beyond a location, for both uniform and general traffic streams. In this model, a traffic stream is divided into a number of cells, and the structure of possible MFR communication chains is clarified. The probability for information to travel to and beyond a vehicle at a certain hop is computed regressively, and the lower bound of the absolute success rate for information to travel beyond a point is defined. Then, based on stochastic modeling, we presented examples of the expected performance of information propagation with respect to different traffic conditions, penetration rates, and transmission ranges.

Although we consider different conditions and measurements for information propagation, the results in this paper are consistent in magnitude with those in (Hartenstein et al., 2001; Yang, 2003). For example, the success rates in Figures 2 and 3 match well with the probabilities for two equipped vehicles to establish connection found in (Hartenstein et al., 2001, Figures 1 and 2). In addition, our result is consistent with the finding in (Yang, 2003, Figure 3.10c) that $7 \mathrm{~km}$ is the maximum information propagation distance. However, unlike these previous studies, the model developed in this paper is analytical and can thus be applied to general conditions.

Study results indicate that information propagation can be quite extensive under high density (congested) conditions. The study also indicates that, when the penetration rate is too low, the instantaneous information propagation is not achievable, and information propagation may only 
be achieved by two-directional information propagation, with the penalty of significant delay, as discussed in (Ziliaskopoulos and Zhang, 2003; Yang, 2003).

The results of this study serve to better understand how well information may propagate through IVC under certain conditions, and to help determine properties of communication devices (in terms of communication range) that potentially could be used to achieve certain performance of such systems. In addition, the models developed in this study can well be extended to help to determine the distance between consecutive road-side stations, deployed as a supplement to the IVC system, to relay information to vehicles in order to achieve better success rates. Another extension to this work involves developing models for information propagation in dynamically changing traffic streams. We believe that such a problem can be tackled in a similar fashion since, compared to other mobile ad hoc networks (Perkins, 2000), the topology change caused by traffic dynamics is relatively small. It would also be desirable to develop an information propagation model coupled with a traffic flow model, such as the Lighthill-Whitham-Richards model (Lighthill and Whitham, 1955; Richards, 1956) and consider information propagation for different road networks.

In this study, we ignored the communication routing algorithms that would be essential for practical ATIS applications. To pursue practically efficient information propagation, specific routing algorithms need to be developed that consider traffic conditions and each vehicle's position,

link, and origin-destination. Yet another practical issue concerns the assumption that the success of transmission is $100 \%$ within the transmission range, performance not generally realizable. However, we believe that the results demonstrate the feasibility of taking advantage of better network connection through inter-vehicle communication in congested traffic to lead to an "internet-on-the-road" that could help make daily stressful driving a more productive journey.

\section{Acknowledgements}

This research was supported, in part, by grants from the National Science Foundation, the U.S. Department of Transportation, and the Division of Research and Innovation, California Department of Transportation. Their support is gratefully acknowledged. We would also like to thank suggestions and comments made by Dr. James Marca and Dr. Craig Ross Rindt of the Institute of Transportation Studies at UC Irvine. The views and results contained herein are the authors' alone and do not necessarily represent the views of these sponsors.

\section{References}

D. E. Boyce, A. M. Kirson, and J. L. Schofer. ADVANCE - the Illinois dynamic navigation and route guidance demonstration program. In I. Catling, editor, Advanced Technology for Road Transport: IVHS and ATT, pages 247-270. Artech House, London, 1994.

L. Briesemeister, L. Schäfers, and G. Hommel. Disseminating messages among highly mobile hosts based on inter-vehicle communication. In IEEE Intelligent Vehicles Symposium, pages 522-527, OCT 2000.

CarTALK 2000. Safe and comfortable driving based upon inter-vehicle communication. http://www.cartalk2000.net/. Accessed November 11, 2003.

W. Feller. An Introduction to Probability Theory and its Applications, volume I. John Willey \& Sons, Inc, New York, 1950.

FleetNet. Internet on the road. http://www.fleetnet.de/. Accessed November 11, 2003. 
F. L. Hall, B. L. Allen, and M. A. Gunter. Empirical analysis of freeway flow-density relationships. Transportation Research Part A, 20:197, 1986.

H. Hartenstein, B. Bochow, A. Ebner, M. Lott, M. Radimirsch, and D. Vollmer. Position-aware ad hoc wireless networks for inter-vehicle communications: the fleetnet project. In Proceedings of the 2nd ACM international symposium on Mobile ad hoc networking 85 computing, pages 259 - 262, Long Beach, CA, USA, 2001.

M. J. Lighthill and G. B. Whitham. On kinematic waves: II. A theory of traffic flow on long crowded roads. Proceedings of the Royal Society of London A, 229(1178):317-345, 1955.

P. K. Munjal, Y. S. Hsu, and R. L. Lawrence. Analysis and validation of lane-drop effects of multilane freeways. Transportation Research, 5:257-266, 1971.

G. F. Newell. A simplified theory of kinematic waves in highway traffic I: General theory. II: Queuing at freeway bottlenecks. III: Multi-destination flows. Transportation Research Part B, 27:281-313, 1993.

C. E. Perkins, editor. Ad Hoc Networking. Addison Wesley Professional, 2000.

P. I. Richards. Shock waves on the highway. Operations Research, 4:42-51, 1956.

M. Rudack, M. Meincke, and M. Lott. On the dynamics of ad hoc networks for inter vehicle communications (IVC). In The 2002 International Conference on Wireless Networks (ICWN'02), Las Vegas, Nevada, USA, 2002.

H. Takagi and L. Kleinrock. Optimal transmission ranges for randomly distributed packet radio terminals. IEEE Transactions on Communications, 32(3):246-257, March 1984.

X. Yang. Assessment of A Self-Organizing Distributed Traffic Information System: Modeling and Simulation. PhD thesis, University of California, Irvine, 2003.

A. K. Ziliaskopoulos and J. Zhang. A zero public infrastructure vehicle based traffic information system. In Transportation Research Board 2003 Annual Meeting CD-ROM, Washington, D.C., 2003. 


\begin{tabular}{ll}
\hline$R$ & transmission range \\
$\rho$ & traffic density \\
$\mu$ & penetration rate of equipped vehicles, $\nu=1-\mu$ \\
$(c, k)$ & vehicle $k$ in cell $c$ \\
$x(c, k)$ & position of vehicle $(c, k)$ \\
$n_{c}$ & the number of vehicles in cell $c$ \\
$\underline{k}$ & $(c-1, \underline{k})$ is the farthest, upstream (in the direction of information propagation) car \\
$\bar{k}$ & inside the transmission range of $(c, k)$ \\
& $(c+1, \underline{k})$ is the farthest, downstream (in the direction of information propagation) car \\
$(c, k ; h)$ & inside the transmission range of $(c, k)$ \\
$P(c, k ; h)$ & the event that vehicle $(c, k)$ is node $h$ of a communication chain \\
$\mathcal{P}(c, l, i ; h)$ & the probability for information to travel to vehicle $(c, k)$ in $h$ hops \\
$P_{1}(c+1, k ; h+1)$ & the joint probability of $(c, i ; h)$ and $(c, l ; h-1)$ \\
& the probability for node $(c+1, k ; h+1)$ to be connected from node $h$ in cell $c$ \\
$P_{2}(c+1, k ; h+1)$ & through a one-hop jump probability for node $(c+1, k ; h+1)$ to be connected from node $h-1$ in cell $c$ \\
& the through a two-hop jump \\
$S(c, k ; h)$ & the success rate for information to travel beyond vehicle $(c, k)$ at $h$ hops \\
$s(c, k)$ & the lower bound of the absolute success rate for information to travel \\
& beyond vehicle $(c, k)$ \\
\hline
\end{tabular}

Table 1: Notations

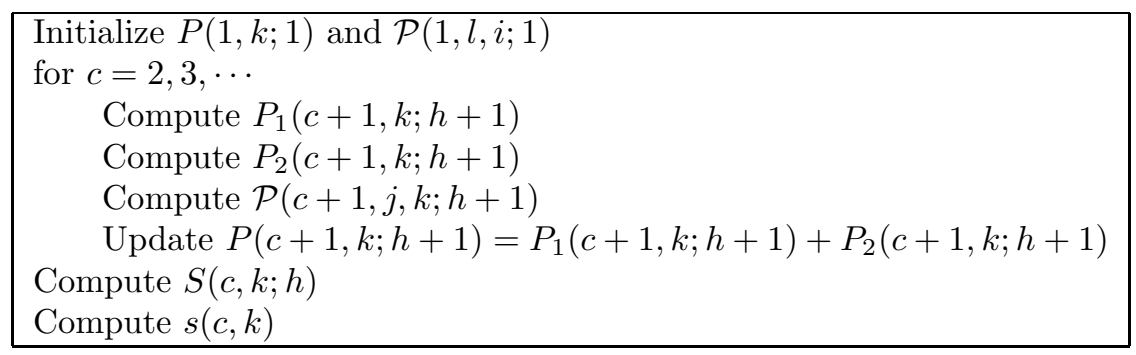

Table 2: The algorithm for computing performance measurements in IVC

\begin{tabular}{|c||c|c|c|c|c|c|c|c|c|c|}
\hline$n$ & $h=1$ & $h=2$ & $h=3$ & $h=4$ & $h=5$ & $h=6$ & $h=7$ & $h=8$ & $h=9$ & $h=10$ \\
\hline$\mu=0.02$ & 114 & 193 & 223 & 243 & 259 & 271 & 281 & 290 & 298 & 304 \\
\hline$\mu=0.05$ & 45 & 76 & 88 & 96 & 102 & 107 & 111 & 114 & 117 & 120 \\
\hline$\mu=0.1$ & 22 & 37 & 43 & 47 & 50 & 52 & 54 & 56 & 57 & 58 \\
\hline$\mu=0.2$ & 11 & 17 & 20 & 22 & 23 & 25 & 25 & 26 & 27 & 28 \\
\hline$\mu=0.3$ & 7 & 11 & 13 & 14 & 15 & 15 & 16 & 16 & 17 & 17 \\
\hline
\end{tabular}

Table 3: Minimum number of vehicles to achieve success rate $90 \%$ at different hops for different penetration rates 


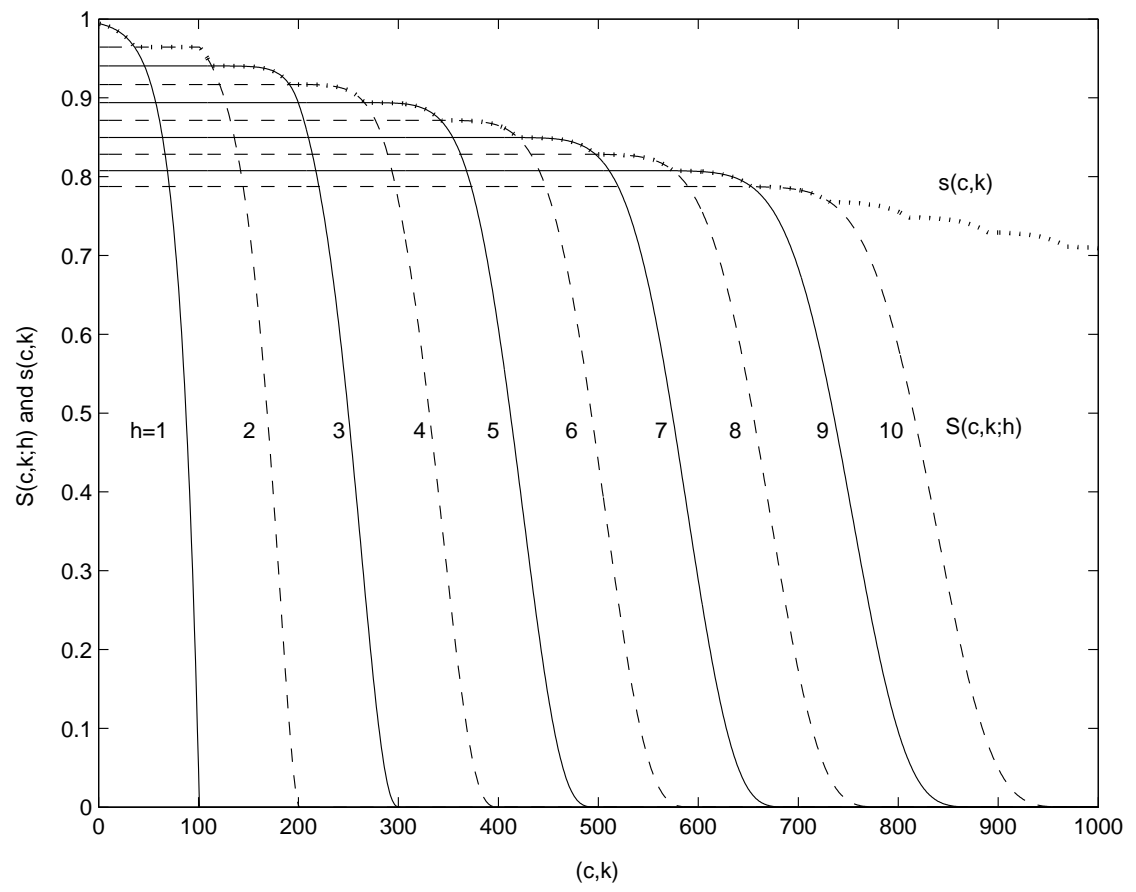

Figure 1: Plots of $S(c, k ; h)$ for $\mu=0.05$ and $n=100$

\begin{tabular}{|c||c|c|c|c|c|c|c|c|c|c|}
\hline$n$ & $h=1$ & $h=2$ & $h=3$ & $h=4$ & $h=5$ & $h=6$ & $h=7$ & $h=8$ & $h=9$ & $h=10$ \\
\hline$s=0.98$ & 38 & 55 & 61 & 65 & 68 & 71 & 73 & 74 & 76 & 77 \\
\hline$s=0.95$ & 29 & 45 & 51 & 55 & 58 & 60 & 62 & 64 & 65 & 67 \\
\hline$s=0.9$ & 22 & 37 & 43 & 47 & 50 & 52 & 54 & 56 & 57 & 58 \\
\hline$s=0.85$ & 19 & 32 & 38 & 42 & 45 & 47 & 49 & 51 & 52 & 53 \\
\hline$s=0.8$ & 16 & 28 & 34 & 38 & 41 & 43 & 45 & 47 & 48 & 50 \\
\hline
\end{tabular}

Table 4: Minimum number of vehicles to achieve different success rates at different hops for penetration rate $10 \%$ 


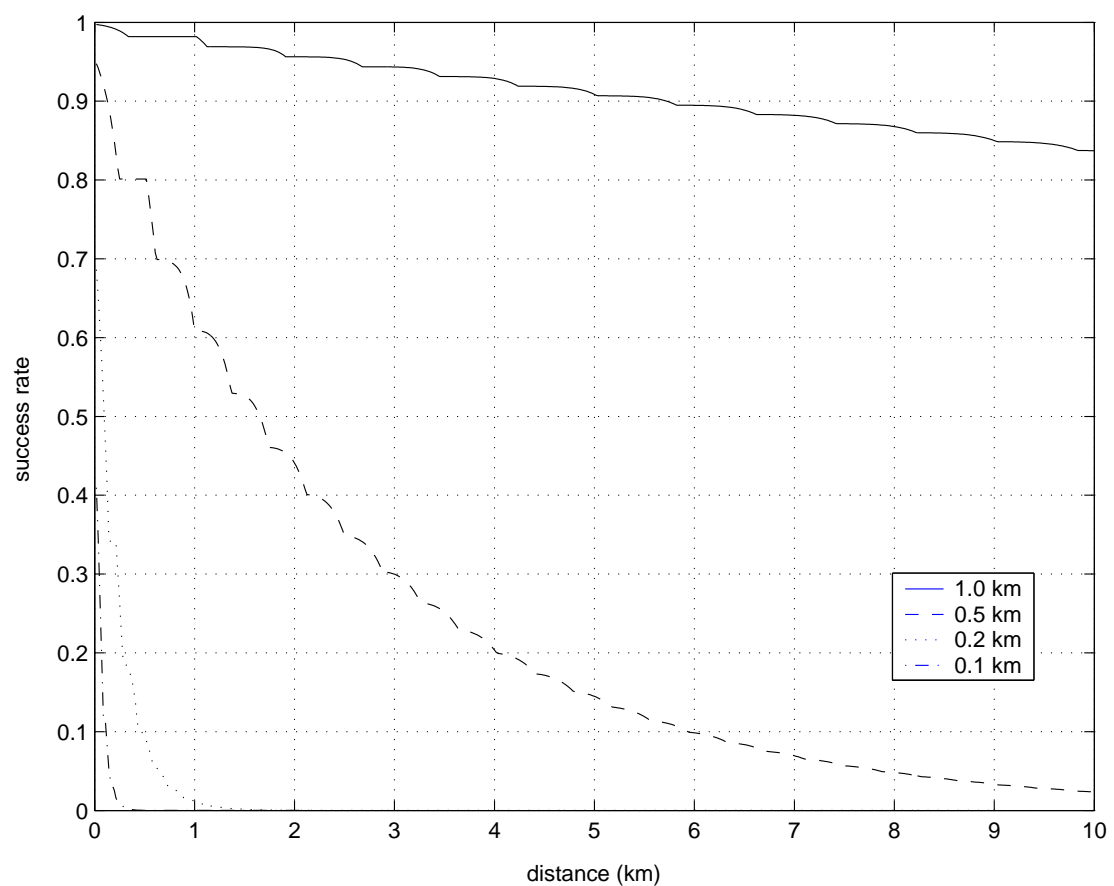

Figure 2: Success rates v.s. transmission ranges: penetration rate $=10 \%$ and four-lane road with density of $56 \mathrm{veh} / \mathrm{km}$ 


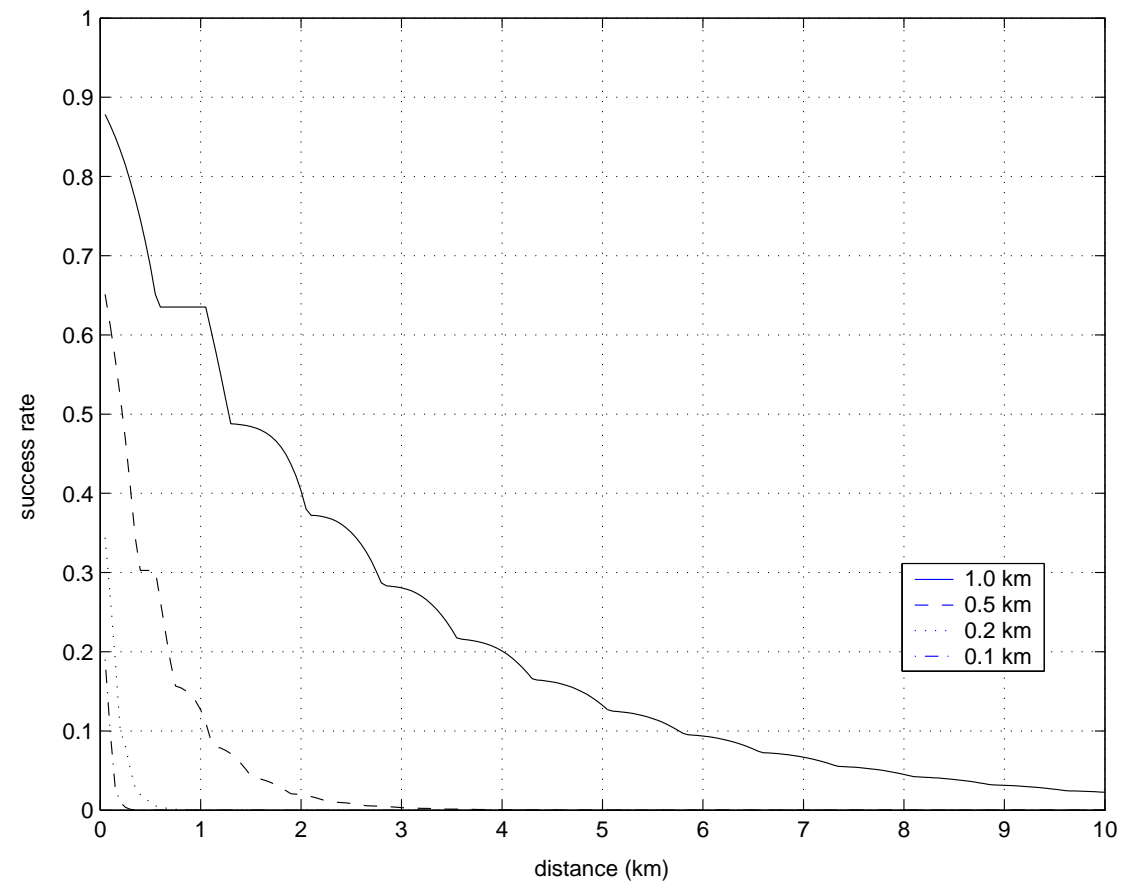

Figure 3: Success rates v.s. transmission ranges: penetration rate $=10 \%$ and four-lane road with density of $20 \mathrm{veh} / \mathrm{km}$

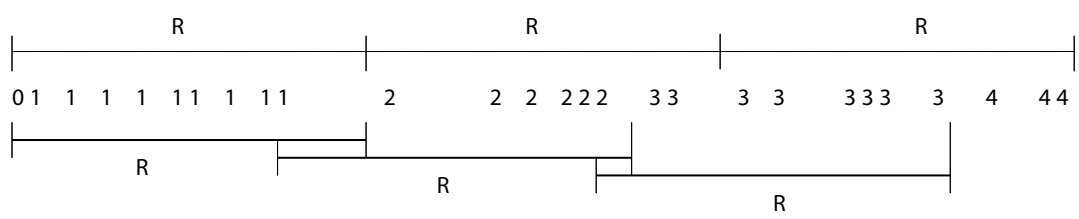

Figure 4: An example of vehicle distribution in general traffic, in which the length of a cell is smaller than $R$. 


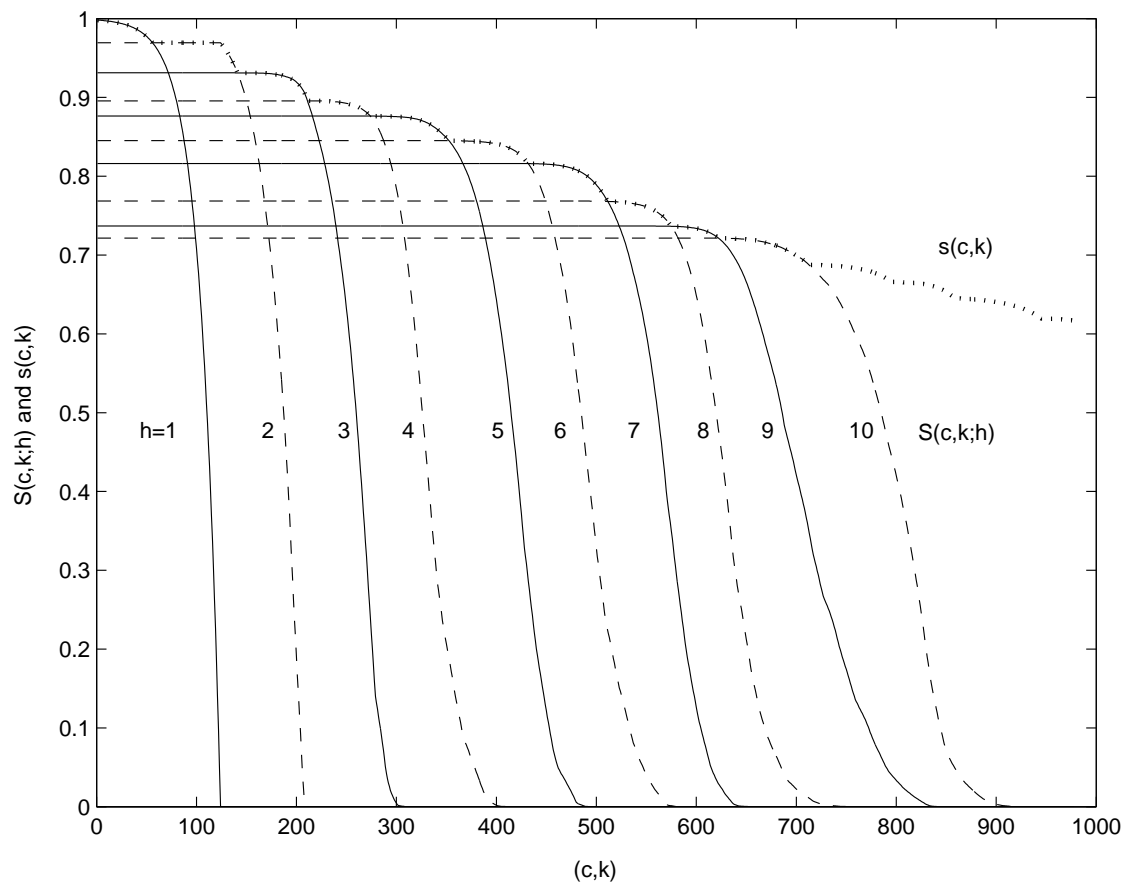

Figure 5: Success rates v.s. vehicle number in a randomly distributed traffic stream.

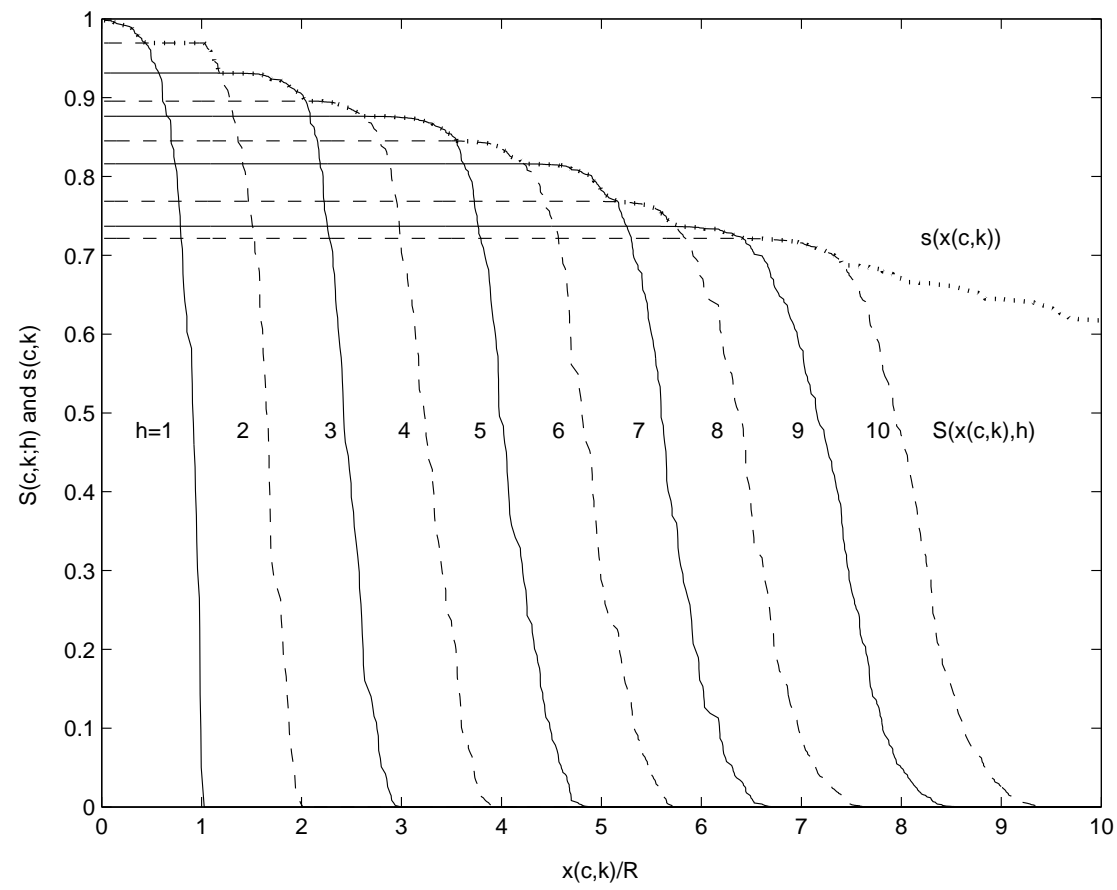

Figure 6: Success rates v.s. vehicle position in a randomly distributed traffic stream. 


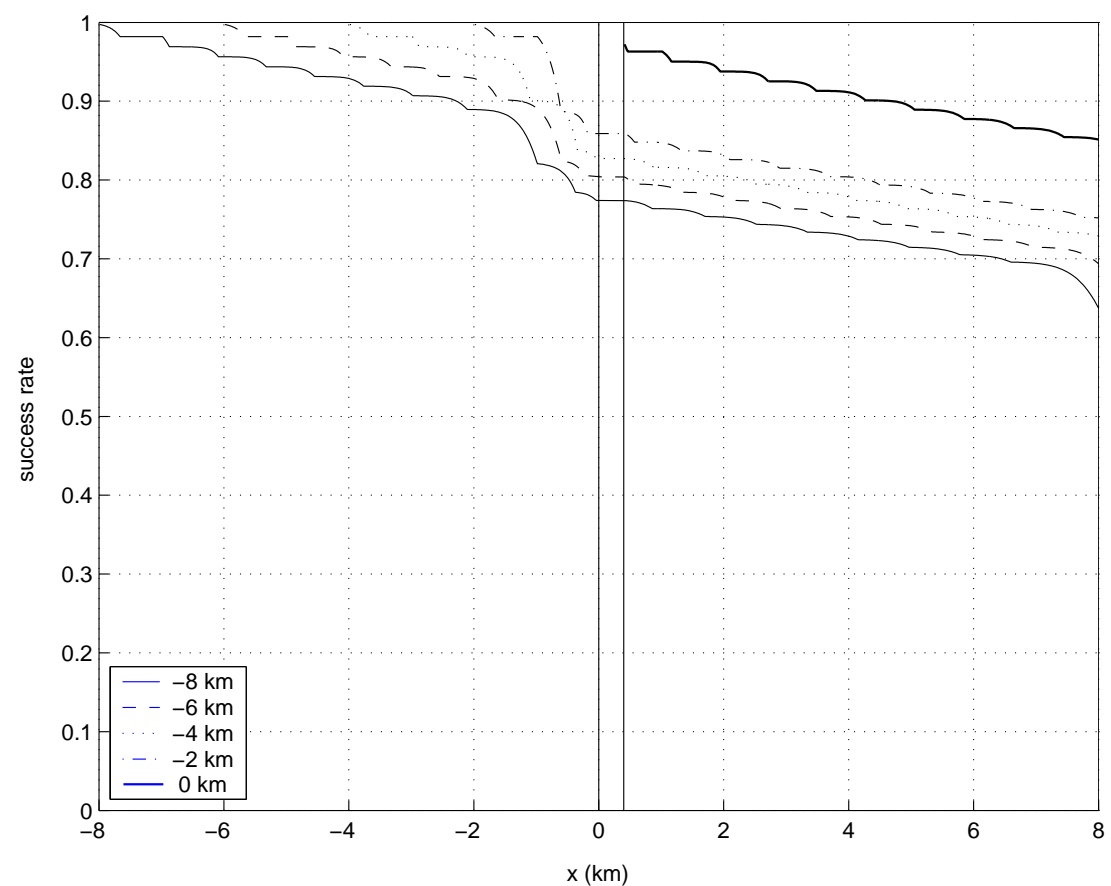

Figure 7: Success rates of information propagation for different locations of information source with a gap at $[0,0.4 \mathrm{~km}]$. 


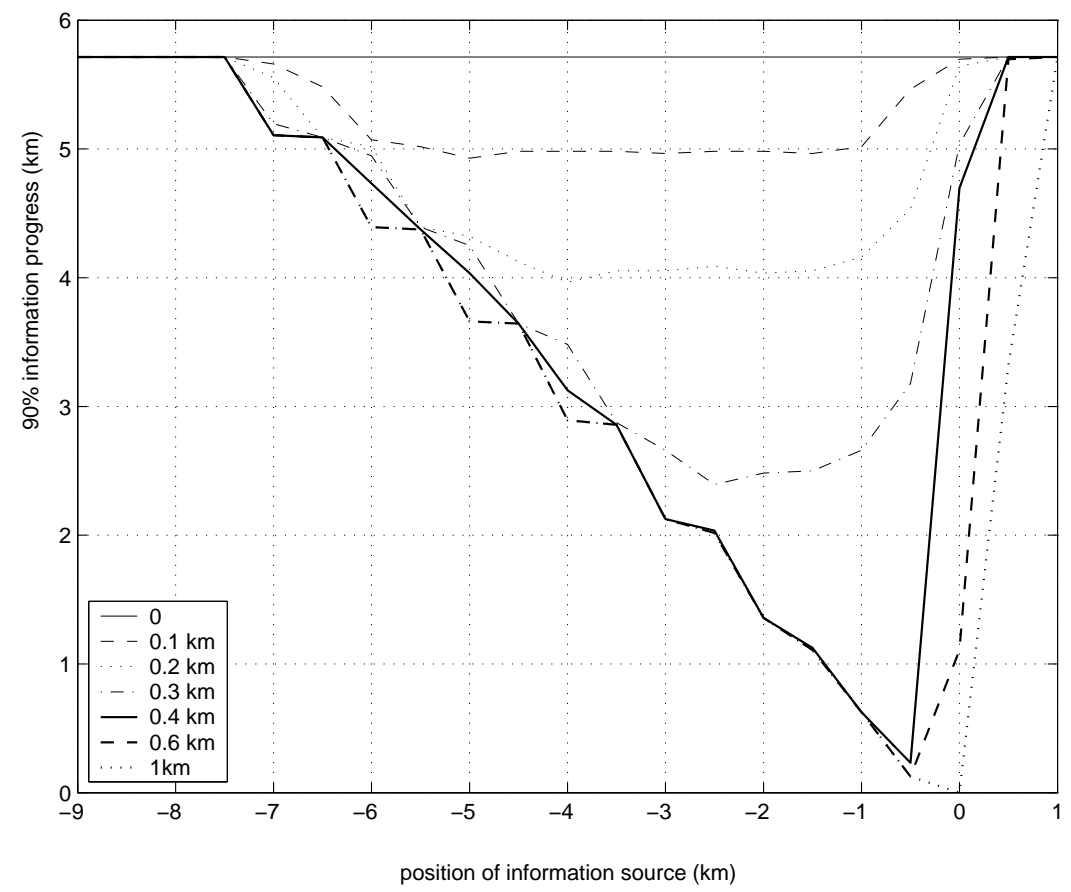

Figure 8: Information progress with $90 \%$ success rate for different sizes of gaps.

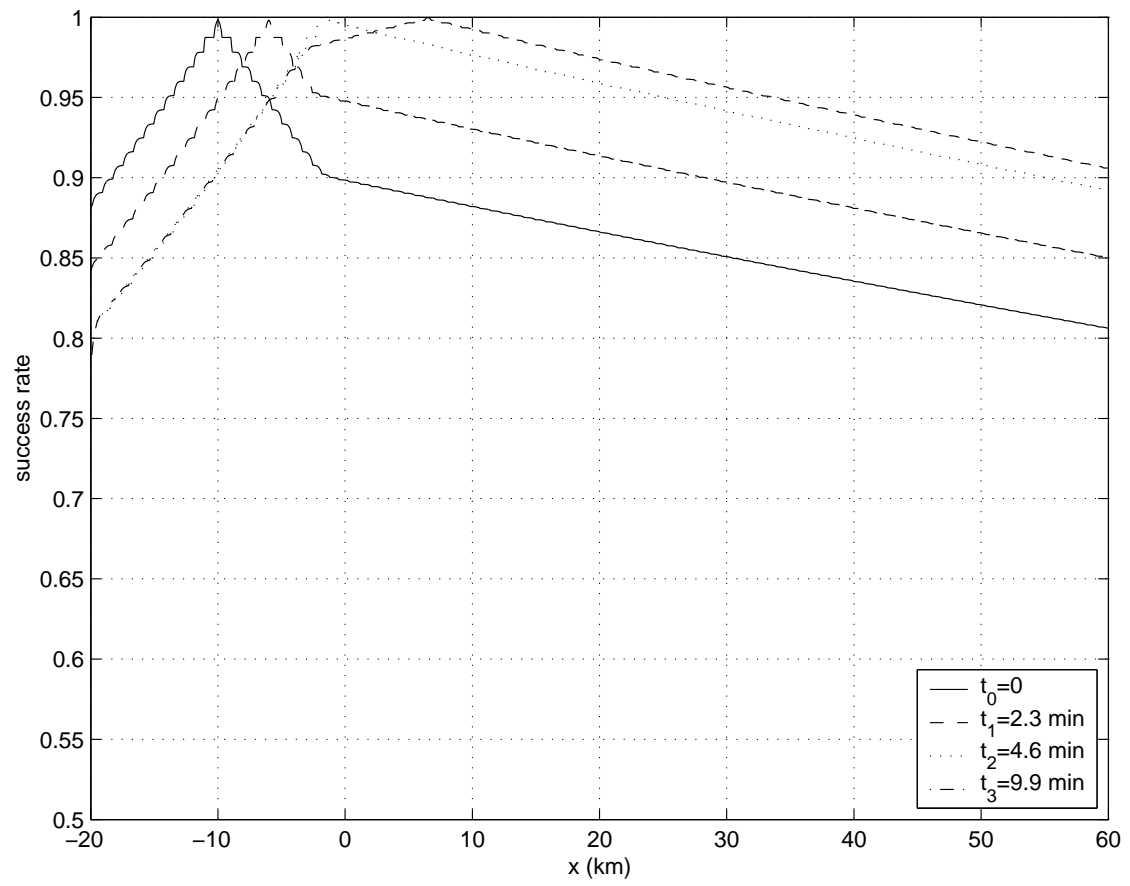

Figure 9: Success rates of information propagation at four instants. 


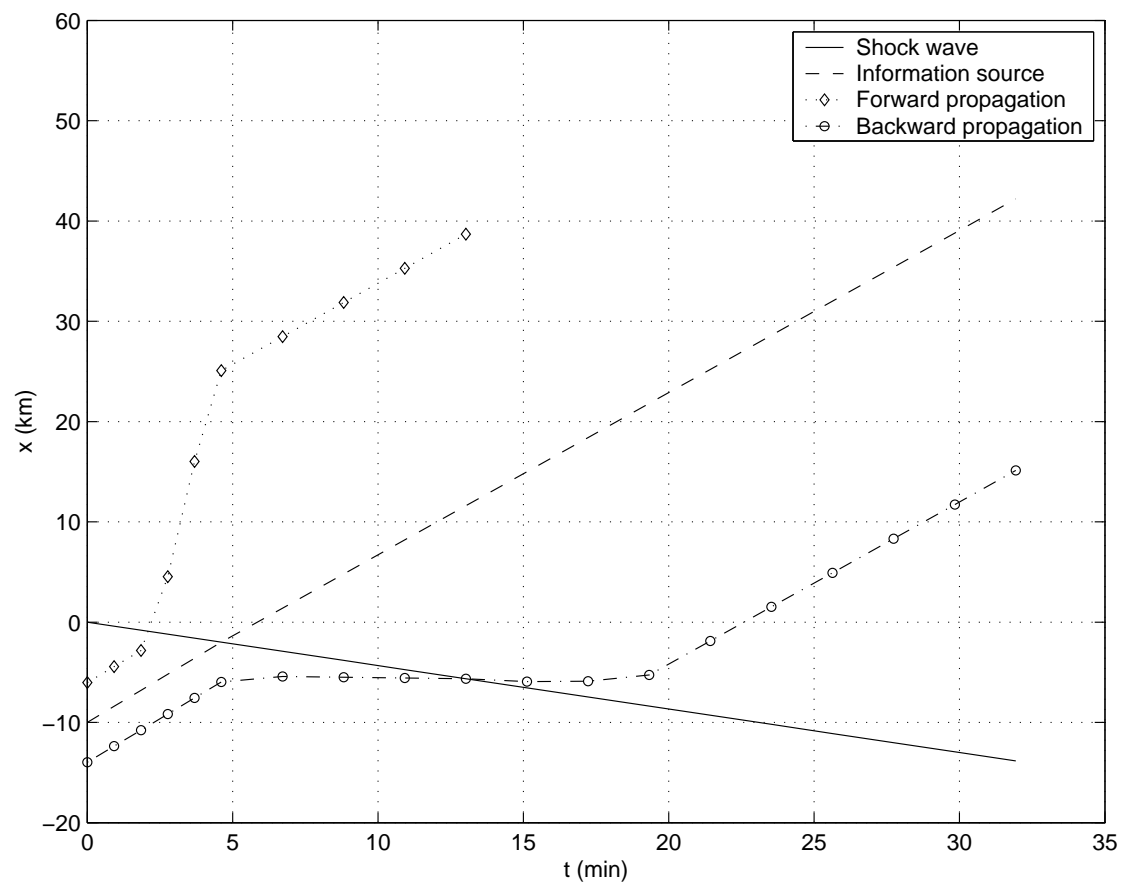

Figure 10: Information progress with $95 \%$ success rate in a shock wave. 